
3 Research Square
Preprints are preliminary reports that have not undergone peer review.
They should not be considered conclusive, used to inform clinical practice, or referenced by the media as validated information.

\title{
High Flow Nasal Oxygen for Acute Type 2 \\ Respiratory Failure: A Systematic Review and Meta- Analysis Protocol
}

\section{Asem Alnajada ( $\sim$ aalnajada01@qub.ac.uk)}

Queen's University Belfast School of Medicine Dentistry and Biomedical Sciences

\section{Bronagh Blackwood}

Wellcome Wolfson Institute for Experimental Medicine, Queen's Univrsity Belfast

\section{Abdulmajeed Almobrad}

Prince Sultan College for Emergency Medical Services, King Saud University

\section{Adeel Akhtar}

Department of Emergency Medicine, Royal Victoria Hospital, Belfast

\section{Murali Shyamsundar}

Wellcome Wolfson Institute for experimental medicine, Queen's University Belfast

\section{Protocol}

Keywords: High flow nasal oxygen, high flow nasal cannula, acute type 2 respiratory failure, acute hypercapnic respiratory failure

Posted Date: March 9th, 2020

DOI: https://doi.org/10.21203/rs.3.rs-16434/v1

License: (c) (1) This work is licensed under a Creative Commons Attribution 4.0 International License. Read Full License 


\section{Abstract}

\section{Background}

Acute Type 2 respiratory failure (AT2RF) is defined by hypercapnia ( $>6 \mathrm{kPa})$ and these patients require ventilatory support in a significant proportion of cases. Non-invasive ventilation (NIV) is effective in preventing escalation to invasive mechanical ventilation (IMV) but has a high failure rate. High flow nasal oxygen (HFNO) is a novel device that integrates humidified air with oxygen at flow rates of up to 60 $\mathrm{L} / \mathrm{min}$. Various additional benefits including carbon-di-oxide $(\mathrm{CO} 2)$ clearance have been attributed to HFNO. This systematic review aims to determine whether the use of HFNO for patients with AT2RF 1) improves arterial $\mathrm{CO} 2(\mathrm{PaCO} 2), 2)$ improves other clinical and patient-centred outcomes and 3) assess any potential harms.

\section{Method}

Searches for relevant studies will be conducted in MEDLINE, EMBASE and the Cochrane Central Register of Controlled Trials, with no language restrictions. In addition, we will search Google Scholar and the references of all articles for any pertinent studies not found in our initial search. With the assistance of a professional librarian, we will develop a systematic search strategy using appropriate keywords and $\mathrm{MeSH}$ terms. We will identify trials that examined the efficacy of HFNO in adult AT2RF patients. We will include randomised trials and observational studies that compared two or more modalities of oxygen delivery that includes high flow nasal oxygen as one of the modes with the comparator being low flow oxygen and/or non-invasive ventilation. The primary outcome is change in $\mathrm{PaCO} 2$ level while other clinical and patient centred outcomes will be collected. Two reviewers will independently assess studies for eligibility, data extraction and quality appraisal. We will conduct a meta-analysis and/or a narrative summary as appropriate.

\section{Discussion}

HFNO is utilised extensively in clinical practice with widening indications of use. The effects of HFNO includes $\mathrm{CO} 2$ clearance and PEEP benefits that may be beneficial to patients with AT2RF. While current publications indicate a beneficial role, no systematic review has attempted to summarise the evidence for this indication. The results of the proposed systematic review will summarise the current evidence to guide practice, guideline development and the design of future trials.

\section{Systematic review registration}

PROSPERO CRD42019148748. (https://www.crd.york.ac.uk/prospero/)

\section{Background}

Acute respiratory failure (ARF) is related to insufficient ventilator pump mechanism and gas exchange (1). ARF is classified as type 1 , which causes hypoxemia (partial pressure of oxygen $(\mathrm{PaO} 2)<8 \mathrm{Kpa}$ ) or 
type 2, which causes hypercapnia (partial pressure of Carbone dioxide oxygen (PaCO2) >6 Kpa) (2).

The significant number of patients presenting with ARF represent a global health burden of epidemic proportions. In the United States of America, ARF cases increased from 502 to 784 cases per 100000 populations from 2001 to 2009. (3). ARF also accounts for nearly 116,000 United Kingdom (UK) hospital admissions for respiratory support per year (4).

Chronic obstructive pulmonary disease (COPD) is the second-most widespread disease in UK, with $1,201,685$ cases reported in 2013 . The overall mortality rate was 29,776 , representing $5.3 \%$ of all UK deaths in 2012 (5). Acute exacerbations of COPD (AECOPD) account for 100,000 admissions annually in England. Of these, around $20 \%$ will present with or develop hypercapnia, an indicator of increased risk of death $(6,7)$. In hospital, mortality in patients with AECOPD is still high, up to $8 \%$ that increases up to $15 \%$ in ICU patients. The one-year mortality in these patients is up to $44 \%$ (8). Adequate treatment of acute type 2 respiratory failure (AT2RF) is essential to prevent mechanical ventilation in these patients to reduce mortality and the demand on critical care resources.

Treatment for AT2RF includes medical therapy such as bronchodilators, diuretics, antibiotics and controlled oxygen therapy aimed at relieving the underlying pathological process such as fluid overload, bronchospasm and infection. Patients will also require ventilatory support that may be non-invasive ventilation (NIV) or invasive mechanical ventilation. Current guidelines recommend the use of NIV $(2,9)$ and current evidence has established the role of NIV in improving arterial oxygenation, hypercapnia and acidosis (10). Additionally, NIV reduces respiratory muscle workload, improves alveolar ventilation, dyspnoea and respiratory rate (10). Early NIV contributes to a reduction in invasive interventions and inhospital mortality in patients with AT2RF (11). However, many studies have highlighted the limitations of NIV. The failure rate of NIV is still up to $40 \%$ with a significant amount of late failure after initial success. The factors leading to NIV failure is multi-factorial including ventilator asynchrony due to mask leak, trigger issues, non-compliance due to claustrophobia, delirium, sputum retention, reduced communication and skin compromise such as, skin necrosis in nasal bridge. Mask discomfort is seen in up to $50 \%$ of patients and skin compromise is seen in up to $20 \%$ of patients. There are also relative medical contraindications including emesis, reduced mentation and reduced access to physiotherapy manoeuvres that limit its use $(12,13)$.

High flow nasal oxygen (HFNO) is a novel respiratory support that integrates humidified air with high flow rate of up to $60 \mathrm{~L} / \mathrm{min}$. Current evidence demonstrates additional benefits from HFNO that include, consistent fractional inspired oxygen (FiO2) delivery, dead space washout, reduced work of breath, comfort and tolerability, ability to communicate, mucous clearance and NIV-like effects which make it a more tolerable method for patients $(14,15)$. In the last 20 years, HFNO was presented as a therapy for neonatal care with a facemask interface $(16,17)$. More recently, HFNO has become a modality of oxygen administration in adult patients with type 1 respiratory failure (18). The device consists of a gas source (air $/ \mathrm{O}_{2}$ ), humidifier, various sizes of circuit tubing, and nasal cannula. Flow rate and $\mathrm{O}_{2}$ concentration can be adjusted based on the patients' condition with flows between $40-60 \mathrm{~L} / \mathrm{min}$. HFNO is not a closed 
system and hence, mouth opening could reduce PEEP effects and increase dilution of inspired oxygen (18).

In the last 10 years, several observational studies have suggested potential benefits of HFNO for AT2RF as demonstrated by improved gas exchange and acidosis. (19-21). In addition, some mechanistic studies have investigated the physiological effects of HFNO for AT2RF with a significant reduction in the respiratory rate and work of breath $(22,23)$. Furthermore, they found that patients were more comfortable with HFNO than NIV. Similar benefit is shown in RCTs with improvements in blood gases in patients with AT2RF (24-26). In addition, some studies evaluated dyspnoea and found that HFNO reduced the work of breath (25). Patients were more comfortable with HFNO than NIV while device noise was shown to be bothersome (27).

\section{Why this review is important}

Adequate respiratory support through controlled oxygen, reduced work of breathing and $\mathrm{CO} 2$ clearance is essential to prevent intubation and invasive ventilation. NIV despite its use has limitations and a high failure rate. HFNO might overcome the limitations of NIV and could be used in these patients as an initial intervention or in patients who do not tolerate NIV. Despite the increase in current literature suggesting benefits from the use of HFNO in AT2RF, current evidence is limited to observational studies and randomized trials on stable type 2 respiratory failure available $(27,28)$ with sparse evidence in patients with AT2RF. Furthermore, there are no clear guidelines for the use of HFNO in AT2RF. Systematic evidence synthesis is required to address this knowledge gap and to guide future developments in this field.

\section{Objectives}

The primary objectives of this systematic review is to determine whether the use of HFNO for patients with AT2RF improves $\mathrm{PaCO}_{2}$ in compare to low flow rate oxygen and/or NIV. Secondary objectives are to examine whether HFNO in patients with AT2RF improves other clinical or patient centred outcomes and to assess any potential harms.

\section{Review question}

In adult patients with AT2RF, is HFNO effective in reducing PaCO2 level and other clinical, mechanistic or patient centred outcomes?

\section{Methods}

\section{Eligibility criteria}

\section{Study types}

Controlled, uncontrolled randomized trials and cohort studies will be included in which HFNO is used as the intervention and low flow oxygen and/or NIV are used as comparators. 


\section{Types of participants}

The participants will be adult population, $\geq 18$ years old, with AT2RF that have been managed by HFNO. AT2RF will be defined as acute respiratory failure with $\mathrm{PaCO} 2$ of $>6 \mathrm{Kpa}(2)$. The study setting will be limited to where the treatment is applied in an acute care setting (emergency department, respiratory ward or critical care units). Studies where HFNO is used as a weaning modality or in peri-operative settings will be excluded or where HFNO is used for patients with reversible conditions like, toxicity or drug overdose will be excluded.

\section{Types of interventions}

HFNO will be defined as application of a flow rate of $\geq 20 \mathrm{~L} / \mathrm{min}$, delivered by nasal cannula. Interventions included will be trials involving HFNO with at least one comparator that can be low flow oxygen (flow rate $<20 \mathrm{~L} / \mathrm{min}$ ), NIV or both.

\section{Outcomes}

The primary outcomes for this review is the Change in $\mathrm{PaCO}_{2}$ after $\mathrm{HFNO}$ application (measured at time points reported by authors).

The secondary outcomes, where available will be

- Respiratory parameters including, $\mathrm{pH}, \mathrm{PaO}_{2}$, dyspnoea score, tidal volume and minute volume (measured at time points reported by authors).

- Mucous clearance (patients' needing mucous clearance before, during or after HFNO application).

- Level of consciousness.

- Patient comfort (patients' tolerance of HFNO compared to other intervention).

- Intubation rate (intubation after HFNO).

- Length of stay in hospital.

- Post-discharge COPD exacerbation rate.

- Readmission rate secondary to AECOPD.

\section{Search strategy}

We will identify trials that examined the efficacy of HFNO in adult acute AT2RF patients. Searches for relevant studies will be conducted on the electronic databases MEDLINE, EMBASE, Cochrane Central Register of Controlled Trials, using no language restrictions. In addition, we will search google scholar and references of all articles for any pertinent studies that were not found in our initial search. With the assistance of a professional librarian, we developed a systematic search strategy using appropriate keywords and MeSH term (see Additional file 1). Medline search strategy will form the basis for the other databases search. 


\section{Data collection and analysis}

\section{Selection of studies}

A systematic review software (Covidence) will be used to store citations and removing duplicates (29). Study screening will undergo different stages by two reviewers independently. First, title and abstract review will be conducted and the studies which does not meet the inclusion criteria will be removed. Any disagreement will be resolved by discussion and referred to a third reviewer. Second, potentially eligible studies will undergo full text review to confirm inclusion. Any disagreement will be resolved through discussion with a third reviewer.

\section{Data extraction}

Two reviewers using a bespoke data extraction form will extract data independently (additional file 2). From eligible studies, information will be extracted on the type and setting of the study, recruitment information, participant characteristics (including age and underlying conditions), inclusion criteria, nature of the intervention(s) in each group (e.g. flow rate and method of delivery), time-points of measurements and outcomes as described above. Any disagreements will be resolved through discussion within the review team. If any of the required data are not available or insufficient from the study publications, they will be requested from the authors of the trial.

\section{Assessment of risk of bias}

For included studies, two reviewers will independently assess risk of bias using the Cochrane Risk of Bias tool for randomized trials and Newcastle-Ottawa scale for cohort studies (see Additional file 2) $(30,31)$. Risk of bias for randomized trials will be assessed for the following domains:

1. Random sequence generation.

2. Allocation concealment.

3. Blinding of participants and personnel.

4. Blinding of outcome assessment.

5. Incomplete outcome data.

6. Selective outcome reporting.

7. Other biases.

Risk of bias for cohort studies will be assessed for the following domains:

Each potential source of bias will be marked as high, low or unclear with justification. We will construct a 'Risk of bias' table in RevMan 5.3 to present the results (32). We will use the assessment of risk of bias to perform sensitivity analyses based on methodological quality, as necessary. To avoid bias within this systematic review, the review will be conducted using this protocol and any variations will be recorded in a specific section of the final published review. 


\section{Measure of effect}

For binary variables, data will be calculated as odds ratios (ORs) with 95\% confidence intervals (Cls). Data for continuous variables will be calculated as mean differences (MDs or as standardised mean differences (SMDs) with $95 \%$ Cls. Where the trial outcomes are sufficiently similar, meta-analysis will be performed and statistical heterogeneity examined between these similar trials. Heterogeneity will be evaluated using the ${ }^{2}$ statistic, with a value of $>50 \%$ possibly implying substantial heterogeneity (31). The pooled estimate will be calculated using the fixed effects model unless there is significant heterogeneity when a random effects model will be used. The outcomes will be presented in forest plot figures. We will undertake two comparisons: trials that compare HFNO versus; low flow rate oxygen and trials that compare HFNO versus NIV. Trials with NIV comparators will be analysed separately due to the established efficacy of NIV in reducing $\mathrm{PCO}_{2}$. When data cannot be meta-analysed, we will present a narrative synthesis and present data in tables.

\section{Unit of analysis}

The unit of analysis will be the patient/participant. We anticipate that all trials will have a parallel group design and thus no adjustment will be necessary for crossover or clustering.

\section{Subgroup analysis and sensitivity analysis}

If sufficient studies are available, we will undertake subgroup analyses for all outcomes against specific patient conditions such as COPD, neuromuscular disorders, interstitial lung disease. We will perform a sensitivity analysis to investigate the effect on the primary outcome of excluding trials with high risk of bias.

\section{Summary of finding tables}

We will assess the quality of the evidence associated with HFNO for AT2RF by using the principles of the GRADE system $(31,33)$. Evidence we be will assessed for methodological quality, directness of evidence, data heterogeneity, precision of effect estimates, and risk of publication bias. We will present the findings using a 'Summary of findings' (SoF) table constructed in RevMan 5.3 (34).

\section{Standards}

Reporting will conform to systematic review and meta-analysis PRISMA checklist preferred reporting items (see Additional file 3) (35). This systematic review has been registered with PROSPERO, an international prospective register of systematic reviews (https://www.crd.york.ac.uk/prospero/).

\section{Discussion}

Despite the increase in studies that suggest benefits from the use of HFNO in AT2RF, including a reduction of $\mathrm{PaCO}_{2}$, increased comfort and improved respiratory parameters, no systematic review to 
date has attempted to summarise the evidence for the use of HFNO for this indication. Furthermore, there are no clear guidelines for the use of HFNO in AT2RF. Systematic evidence synthesis is required to address this knowledge gap. To the best of our knowledge, no review has investigated the benefits of HFNO for acute T2RF. It is expected that the review of current evidence will inform practice and future trials.

\section{Abbreviations}

ARF: Acute respiratory failure

AT2RF: Acute hypercapnic respiratory failure

COPD: Chronic obstructive pulmonary diseases

AECOPD: Acute exacerbation of chronic obstructive pulmonary disease

HFNO: High flow nasal oxygen

NIV: Nom-invasive ventilation

PaO2: Partial pressure of oxygen

PaC02: Partial pressure of carbon dioxide

CENTRAL: Cochrane Central Register of Controlled Trials

\section{Declarations}

Ethics approval and consent to participate

Not applicable

Consent for publication

Not applicable

Availability of data and materials

Not applicable

Competing interests

None

Funding 
The work is funded and sponsored by studentship provided by King Saud University, Saudi Arabia.

Authors' contributions

AAA and MS conceived the idea for the review. AAA drafted the protocol under the supervision of MS and BB. AMA and AA reviewed the manuscript. All authors approved the final manuscript. The funder, sponsor and the institutions had no involvement in developing the protocol.

Acknowledgements

The authors extend their appreciation to the Deanship of Scientific Research at King Saud University for supporting the Research Group.

The authors wish to thank the Queen's University Belfast library team especially Richard Fallis for their support in developing the search strategy.

\section{References}

1. Roussos C. and Koutsoukou A. Respiratory failure. Eur Respir J. 2003;22(47):3s-14s.

2. Rochwerg B, Brozek J, Brochard L, Elliott MW, Hess D, Hill NS, et al. Official ERS/ATS clinical practice guidelines: Noninvasive ventilation for acute respiratory failure. Eur Respir J. 2017;50(2):1602426.

3. Stefan M, Shieh M, Pekow P, Rothberg M, Steingrub J, Lagu T, et al. Epidemiology and outcomes of acute respiratory failure in the United States, 2001 to 2009: A national survey. J Hosp Med. 2013;8(2):76-82.

4. Harrison D. Number of mechanically ventilated patients during 2012. 2014 [ 2019-7-26];;7]. Available from: https://www.icnarc.org/DataServices/.../194bd654-e14a-e411-a65b-d48564544b14

5. Salciccioli J, Marshall D, Shalhoub J, Maruthappu M, De Carlo G, Chung K. Respiratory disease mortality in the United Kingdom compared with EU15+ countries in 1985-2015: Observational study. BMJ. 2018;363:k4680.

6. Plant P. One year period prevalence study of respiratory acidosis in acute exacerbations of COPD: Implications for the provision of non-invasive ventilation and oxygen administration. Thorax. 2000;55(7):550-554.

7. Roberts C, Stone R, Buckingham R, Pursey N, Lowe D. Acidosis, non-invasive ventilation and mortality in hospitalised COPD exacerbations. Thorax. 2010;66(1):43-48.

8. Scala R. and Heunks L. Highlights in acute respiratory failure. Eur Respir Rev. 2018;27(147):8-11.

9. Basarik B. BTS/ICS Guideline for the Ventilatory Management of Acute Hypercapnic Respiratory Failure in Adults. Turkish J Med Surg Intensive Care. 2016;7(1):39-40.

10. Nicolini A, Ferrera L, Santo M, Ferrari-Bravo M, Del Forno M, Sclifo F. Noninvasive ventilation for hypercapnic exacerbation of chronic obstructive pulmonary disease: Factors related to noninvasive ventilation failure. Polish Archives of Internal Medicine. 2014;124(10):525-531. 
11. Plant $\mathrm{P}$, Owen J, Elliott $\mathrm{M}$. Early use of non-invasive ventilation for acute exacerbations of chronic obstructive pulmonary disease on general respiratory wards: A multicentre randomised controlled trial. The Lancet. 2000;355(9219):1931-1935.

12. Ngandu H, Gale N, Hopkinson J. Experiences of noninvasive ventilation in adults with hypercapnic respiratory failure: A review of evidence. Eur Respir Rev. 2016;25(142):451-471.

13. Gay PC. Complications of noninvasive ventilation in acute care. Respir Care. 2009;54(2):246-257.

14. Ischaki E, Pantazopoulos I, Zakynthinos S. Nasal high flow therapy: a novel treatment rather than a more expensive oxygen device. Eur Respir Rev. 2017;26(145):170028.

15. Möller W, Feng S, Domanski U, Franke K, Celik G, Bartenstein P. Nasal high flow reduces dead space. Journal of Applied Physiology. 2019;122(1985):191-7.

16. Roca O, Hernandez G, Diaz-Lobato S, Carratala JM, Gutierrez RM, Masclans JR, et al. Current evidence for the effectiveness of heated and humidified high flow nasal cannula supportive therapy in adult patients with respiratory failure. Crit Care. 2016;20(1):109.

17. Helviz Y, Einav S. A Systematic Review of the High-flow Nasal Cannula for Adult Patients. Crit Care. 2018;22(1):71.

18. Ashraf-kashani N, Kumar R. High-flow nasal oxygen therapy. BJA Education. 2017;17(2):63-67.

19. Kim E, Lee H, Kim S, Park J, Lee Y, Park J, et al. Effectiveness of high-flow nasal cannula oxygen therapy for acute respiratory failure with hypercapnia. J Thorac Dis. 2018;10(2):882-8.

20. Bräunlich J, Wirtz H. Nasal high-flow in acute hypercapnic exacerbation of COPD. Int J COPD. 2018;13:3895-7.

21. Yuste M, Moreno O, Narbona S, Acosta F, Peñas L, Colmenero M. Efficacy and safety of high-flow nasal cannula oxygen therapy in moderate acute hypercapnic respiratory failure. Rev Bras Ter Intensiva. 2019;31(2):156-63.

22. Diziura $M$, Chweich $H$, Hill N. Physiological effect of high-flow nasal cannula on respiratory pattern and work of breathing in severe COPD patients. AJRCCM.2017;195(9):3-5.

23. Longhini F, Pisani L, Lungu R, Comellini V, Bruni A, Garofalo E, et al. High-Flow Oxygen Therapy After Noninvasive Ventilation Interruption in Patients Recovering From Hypercapnic Acute Respiratory Failure: A Physiological Crossover Trial. Crit Care Med. 2019;47(6):e506-11.

24. Doshi P, Whittle J, Bublewicz M, Kearney J, Ashe T, Graham R. high-velocity nasal insufflation in the treatment of respiratory faliure: A randomized clinical trial. Ann Emerg Med. 2018;72(1): 83e5-73.

25. Lee M, Choi J, Park B, Kim B, Lee S, Kim SH, et al. High flow nasal cannulae oxygen therapy in acutemoderate hypercapnic respiratory failure. Clin Respir J. 2018;12(6):2046-56.

26. Francois J, Claire M, Guislaine L, Adrien W, Thibaut D, Gilles C. High flow nasal oxygenotherapy for management acute hypercapnic respiratory failure in emergency departement. Ann Intensive Care. 2018;8(1).

27. Mckinstry S, Pilcher J, Bardsley G, Berry J, suvande H, Braithwaite I, et al. Nasal high fl ow therapy and PtCO 2 in stable COPD : A randomized controlled cross-over trial. Respirology. 2018;378-84. 
28. Covidence systematic review software, Veritas Health Innovation, Melbourne, Australia. Available at www.covidence.org

29. Wells GA, Shea B, O'Connell, Peterson J, Welch V, Losos M, et al. The Ottawa Hospital. [internet]. 2019 [cited 2019 Aug 21]; [one screen]. Avalible from:

http://www.ohri.ca/programs/clinical_epidemiology/oxford.asp

30. Higgins JPT, Altman DG, Gøtzsche PC, Jüni P, Moher D, Oxman AD, et al. The Cochrane Collaboration's tool for assessing risk of bias in randomised trials. BMJ. 2011;343(7829):1-9.

31. L Cochrane Community. 2019 [2019 Aug 21]; [ one screen].Available from: https://community.cochrane.org/help/tools-and-software/revman-web

32. Guyatt G, Oxman A, Kunz R, Falck-Ytter Y, Vist G, Liberati A, et al. Rating quality of evidence and strength of recommendations: going from evidence to recommendations. BMJ. 2008;336:1049-51.

33. Review Manager (RevMan) [Computer program]. Version 5.3. Copenhagen: The Nordic Cochrane Centre, The Cochrane Collaboration, 2014.

34. Kamioka H. Preferred reporting items for systematic review and meta-analysis protocols (prisma-p) 2015 statement. Japanese Pharmacol Ther. 2019;47(8):1177-85.

\section{Supplementary Files}

This is a list of supplementary files associated with this preprint. Click to download.

- Additionalfile2.docx

- Additionalfile3.docx

- Additionalfile4.pdf

- Additionalfile1.doc 